\title{
DOCUMENT SIMILARITY BASED ON CONCEPT TREE DISTANCE
}

\author{
BY \\ Praveen Lakkaraju \\ B.E., Information Technology, \\ Chaitanya Bharathi Institute of Technology, Hyderabad - Osmania University, \\ India - May 2004
}

Master's Thesis

Submitted to the Department of Electrical Engineering and Computer Science and the Faculty of the Graduate School of the University of Kansas in partial fulfillment of the requirements for the degree of Master's of Science in Computer Science

$\begin{array}{cl}\text { Chairperson } & \text { Prof. Susan Gauch } \\ \text { Committee members } & \text { Dr. John Gauch } \\ & \text { Dr. Donna Haverkamp }\end{array}$

Date defended: 
The Thesis Committee for Praveen Lakkaraju certifies

That this is the approved Version of the following thesis:

DOCUMENT SIMILARITY BASED ON CONCEPT TREE DISTANCE

Thesis Committee

Chairperson: Prof. Susan Gauch

Dr. John Gauch

Dr. Donna Haverkamp

Date Approved: 


\section{Acknowledgements}

I would like to thank my advisor Dr. Susan Gauch for her guidance and support in this work and also all through my stint at the University of Kansas. Her patience and constant encouragement helped me to cultivate the great habit of hard work. I learnt a lot from the numerous discussions that I had with her. They helped me grow as a person and I am very fortunate to be associated with such a wonderful teacher.

I would like to thank Dr. John Gauch for serving on my thesis committee and also for giving me a chance to work on an interesting bioinformatics project with him during my second semester. I would also like to thank Dr. Donna Haverkamp for serving on my thesis committee.

I am very grateful to my parents for their constant encouragement and support in realizing my dreams. Finally, I would like to thank the system administrators at ITTC for their timely help while I was working on this thesis. 


\begin{abstract}
The Web is fast moving from an era of search engines to an era of discovery engines. Discovery engines help you find things that you never knew existed or did not know how to ask for. One of the ways this can be done is by automatically computing and displaying objects that are similar to the object in which the user is currently expressing interest. In this paper, we present a new approach to compute interdocument similarity that is based on a tree-matching algorithm. We represent each document as a concept tree using the concept associations obtained from a classifier. We make use of a tree-matching algorithm called the tree edit distance to compute similarities between these concept trees. Experiments on a subset of documents from the CiteSeer collection showed that our algorithm performed better than the document similarity based on the traditional vector space model.
\end{abstract}




\section{TABLE OF CONTENTS}

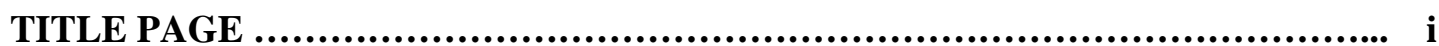

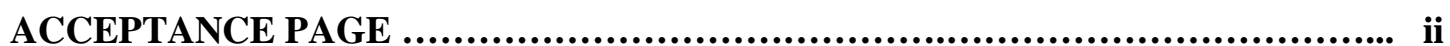

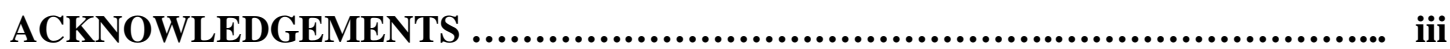

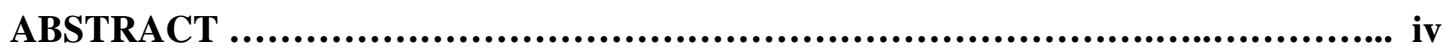

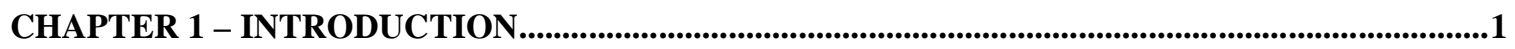

1.1 MOTIVATION

1.2 GOALS

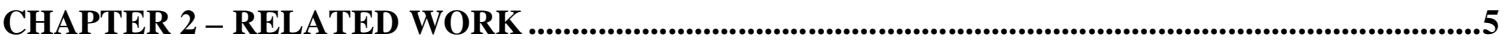

2.1 Document Similarity BaSEd ON Vector SPACE Model ……..................................................

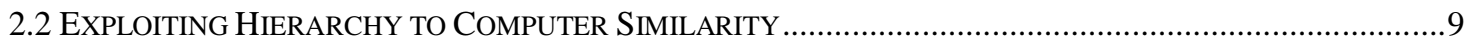

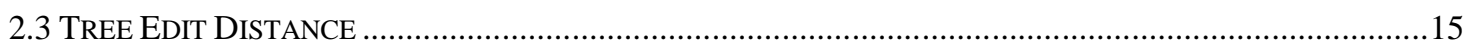

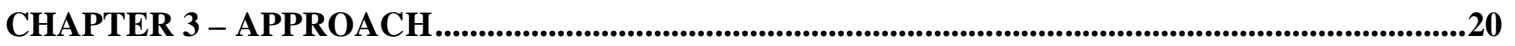

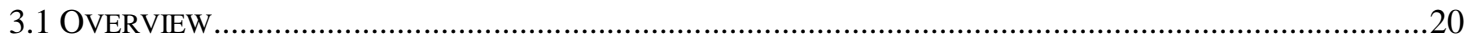

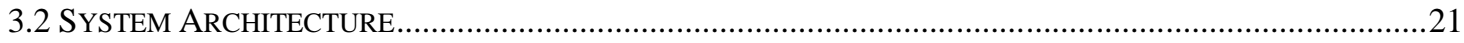

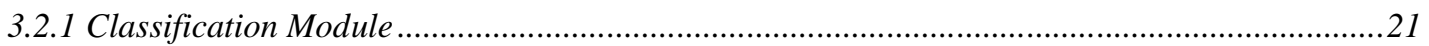

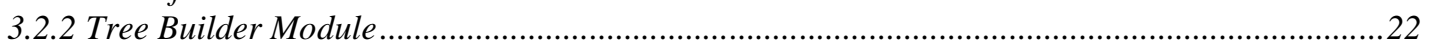

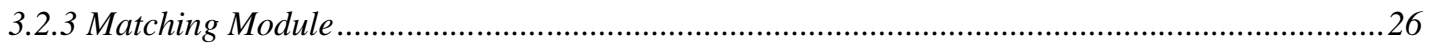

CHAPTER 4 - EVALUATION METHODOLOGY AND RESULTS ......................................................29

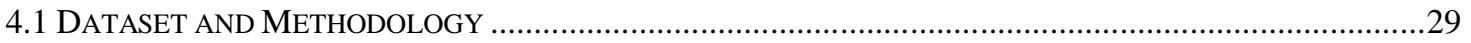

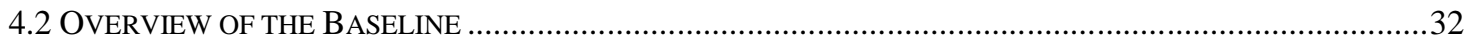

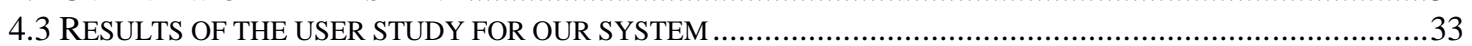

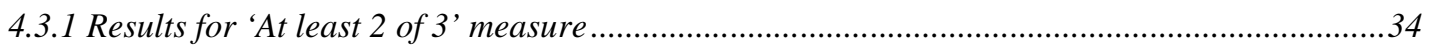

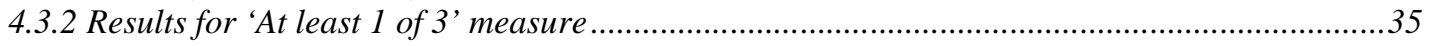

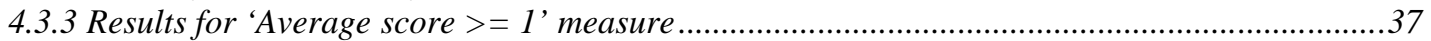

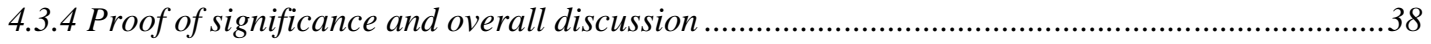

CHAPTER 5 - CONCLUSIONS AND FUTURE WORK ...............................................................................41

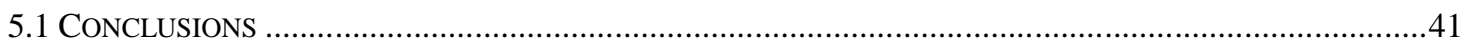

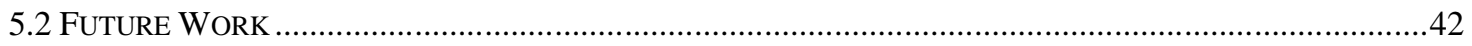

REFERENCES 


\section{Chapter 1 - Introduction}

\subsection{Motivation}

The Web is fast moving from an era of search to an era of discovery. 'Search is what you do when you're looking for something. Discovery is when something wonderful that you did not know existed, or did not know how to ask for, finds you' [12]. One of the ways in which applications help users discover things is by showing them objects similar to those that the user is currently interested in. Examples of this are the 'more like this' or 'similar pages' links that accompany the search results of some of the Web search engines, product recommendations by e-commerce Websites likes Amazon, document recommendations by digital libraries like CiteSeer [11] and related News links on some of the News Websites. Even some of the video sharing applications, e.g., YouTube, show a list of videos that are related to the video currently being viewed by its users. These recommendations or related objects can be obtained in several different ways. One of the ways is to find similar objects from the existing database.

Similarity can be determined by various aspects of objects depending on the domain and the appropriate definition of similarity for that domain. In the case of collaborative filtering based recommender systems, users are clustered based on their previous actions such as purchase history and browsing history. Using this information, recommendations are produced for a given user, which consist of 
products that other similar users have liked or purchased. Document similarity can be computed by matching the text within the documents.

One of the most widely used techniques to calculate document similarity is based on the vector space model's cosine similarity measure. In this method, the documents are considered to be part of the vector space and each document is treated as vector of words. The value of the dot product between two such vectors is used as a measure of similarity. The advantage of this approach is its simplicity of implementation. However, these vectors can be extremely sparse. This approach also assumes that different words in the document are semantically independent and in the similarity calculation, a given word in one document must match to the same word in the other document. It does not take into consideration the existence of synonyms for different words. Approaches such as Latent Semantic Indexing [6] have been proposed to overcome these problems. Some of the other methods for computing document similarity that are based on matching the text within the documents are discussed in Chapter 2.

To handle vocabulary ambiguity, we could use text classifiers such as Rocchio and SVM to identify the most important concepts for each document. Documents could thus be represented as vectors of concepts rather than vectors of keywords, and the cosine similarity measure can be used on these concept vectors to compute the similarity between any two documents. Because we match documents based on the concepts they belong to instead of their actual text, this overcomes some of the 
problems discussed above. However, matching documents purely based on their concept vectors might not give the best results. The concept space is often represented as a hierarchical tree structure, or taxonomy. Representing the concepts as a vector loses the parent-child relationships between the concepts in the taxonomy that could be used while computing the similarity between two documents. In our approach, we exploit the hierarchical relationships between the concepts to construct what we call a 'concept tree' to represent each document. Any one of the several tree-matching algorithms available in the literature can be applied to compute the similarity between two such 'concept trees'. In this work, we make use of a widely used tree-matching algorithm called the tree edit distance algorithm to match our concept trees.

The problem of matching trees finds its application in a wide range of areas such as computational biology, compiler optimization, computer vision, XML databases and natural language processing. One of the popular methods used in comparing trees is the tree edit distance algorithm. In general, trees can be transformed by application of elementary edit operations, which have a certain cost associated with them. The edit distance between two trees is defined in [7] as 'the cost of a least-cost sequence of elementary edit operations, or the length of a shortest sequence of elementary edit operations, that allows one to transform one of the trees into the other'. We apply a simplified version of this algorithm to compute similarity between concept trees. Our approach is discussed in detail in Chapter 3.

\subsection{Goals}

The goals of this thesis are to: 
1. Come up with a method to construct concept trees to represent documents

2. Come up with an algorithm to match documents based on concept trees

3. Demonstrate that this approach is more accurate than traditional vector space similarity.

The rest of the document is organized as follows. The related works in this subject area are discussed in Chapter 2. Chapter 3 discusses our approach in detail. In Chapter 4, we discuss the results of our evaluation experiments and finally in Chapter 5, we present our conclusions and future work. 


\section{Chapter 2 - Related Work}

The literature related to computing similarity between data objects can be divided into two broad categories. The first uses the information present within the data objects like the semantic information in case of text documents while computing similarity. The second makes use of the extraneous information about the data objects like the categories they belong to and the structural context in which they occur while computing similarity. Our work falls into the second category. In the next section, we review some of the work in the literature that makes use of the first approach discussed above. In Section 2.2, we review some of the work related to the second approach discussed above. In Section 2.3, we give some background of the tree edit distance algorithm and also review some work that used this algorithm to compute similarity.

\subsection{Document Similarity based on Vector Space Model}

Lot of researchers made use of keyword vectors to compute similarity between documents. The applications range from computing similarity between short text snippets like search queries [21] to proposing special indexing techniques to answer similarity search queries [3]. We discuss some of their work in this section.

In [21], the authors discuss an approach for computing similarity between short text snippets. They demonstrate that using the traditional cosine measure would not give good results in case of short text snippets like search queries. In their approach, they use a search engine over text corpora to come up with a set of top ranked documents 
for a given text snippet. They then compute the word vectors for each of these documents that contain the top $m$ words based on their TFIDF weights. For a given text snippet $x$, they obtain the query expansion of $x$, by computing the $\mathrm{L}_{2}$ normalization of the centroids of these vectors. This is represented as $\mathrm{QE}(\mathrm{x})$. In order to improve efficiency, they only consider the 'contextually descriptive text snippet' for each document that is commonly generated by Web search engines to build their vectors. They make use of 1,000 characters in a token-delimited window centered on the original query terms in the original text. The kernel function they use to compute the semantic similarity between any two text snippets $x$ and $y$ is shown in the equation below.

$$
\mathrm{K}(\mathrm{x}, \mathrm{y})=\mathrm{QE}(\mathrm{x}) \cdot \mathrm{QE}(\mathrm{y})
$$

They evaluated their approach in two phases. In the first phase they examined several text snippet pairs to determine the similarity score given by their approach, the traditional cosine measure, and the set overlap measure proposed by Fitzpatrick and Dent. They also performed a user study by using their method in a query suggestion system where new queries are suggested to users based on their current query. In both the cases, they observed that their kernel is an effective measure of similarity for short text snippets and works well even when the short texts being considered have no common terms. 
In [24], the authors use the Earth Mover's Distance measure to compute document similarity. The Earth Mover's distance or EMD [17] computes the distance between two distributions, which are represented by signatures. The signatures are sets of weighted features that capture the distributions. The EMD is defined as the minimum amount of work needed to change one signature into the other. The authors in [24] talk about how two different words in two documents can have the same meaning in case of synonyms and how the same word can mean two different things in two different contexts. Keeping these issues in mind they first compute the semantic distance between words using an electronic lexical database, Wordnet. They then apply the EMD algorithm to measure document similarity with a many to many matching between words. In their context, the distribution is the distribution of words in the documents. They represent each document as a set of ordered pairs on the words in the document and their corresponding TFIDF weights. They also have a distance matrix that represents the semantic distance between the words in the two documents. Then they construct a weighted graph whose vertices are the words in the two documents and edges are the semantic distances between these words. The similarity value between two documents is the minimum cost of this weighted graph. This EMD based approach is similar to our approach as it tries to compute the minimum amount of work needed to change one signature to other. But we represent documents as concept trees and apply a tree transformation algorithm to compute similarity. 
In [25], the authors discuss their approach to compute similarity between heterogeneous data objects by making use of their interrelationships. They make use of a Unified Relationship Matrix or URM to represent these data objects and their interrelationships. The similarity between two data objects is obtained by iteratively computing over their URM. For example, Web search queries and Web pages can be considered as heterogeneous data objects. The similarity between two queries can be reinforced by the similarity between the Web pages they relate to. In the same way, the similarity between two Web pages is reinforced by the queries for which they are retrieved and also the similarity between other Web pages to which they relate. They evaluated their approach by making use of query log data of a real world Web search engine that contained 62.5 million queries along with the click-through data of their resulting Web pages. They observed statistically significant improvement over the traditional approach based on TFIDF and also SimRank [13], by making use of their SimFusion algorithm.

An interesting way to solve the problem of similarity search is presented in [3]. They describe the process of building efficient indices to answer similarity search queries. Their indexing schema called LSH Forest, makes use of the well-known technique of locality-sensitive hashing (LSH). In locality-sensitive hashing, hash functions are chosen such that the probability of similar objects hashing to the same bucket is much more than that for objects that are far apart. The key here is to choose the hash functions such that "good" collisions occur. In order to find the objects that are similar to a given object, we simply can find the bucket it hashes to and retrieve all 
the objects in that bucket. Then any kind of similarity metric can be applied to these objects to find the best match. This approach would avoid the need to match against the complete dataset. In [3], the authors point out that the problems with traditional LSH are that the parameters must be tuned to suit each domain and also the storage requirements are proportional to the size of the corpus. Their indexing schema called the LSH Forest overcomes these problems. They evaluated their approach in the context of text domain using the Trec and Reuters collections. The showed that their approach provided better accuracy than the basic LSH scheme and also eliminates the need for various data-dependent tuning.

\subsection{Exploiting Hierarchy to Computer Similarity}

There has been a lot of work done concerning finding similar objects within a given domain by modeling or visualizing the objects and the domain as graphs, trees, or some kind of a hierarchical structure. We review some of this work in this section.

In [8], the authors present an approach to compute similarity by exploiting the hierarchical structure in any given domain. The motivation behind their work is very similar to the motivation behind our work. They consider each object to be a collection of elements. Instead of just considering the two objects whose similarity they are trying to compute as bags of elements, they make use of the underlying hierarchical domain structure to come up with more intuitive similarity measures. They start off by first presenting modified versions of the traditional cosine similarity measure. They then extend these to solve the problem of multiple occurrences of elements. In all their measures, they consider the elements as being the leaves of the 
hierarchy. Our approach does not impose any such restrictions on the elements in an object and they can be at any level of the hierarchy.

In their first measure, called the Generalized Cosine Similarity Measure or GCSM, they take into account the depth of the lowest common ancestor (LCA) in the hierarchy for any two elements to assign a value to the dot product of their unit vectors. This is different from the traditional cosine similarity measure where the value of the dot product between two different unit vectors would be zero. So for any two elements $l_{1}$ and $l_{2}$, they define:

$$
\overrightarrow{l_{1}} \cdot \overrightarrow{l_{2}}=\frac{2 * \operatorname{depth}\left(L C A_{U}\left(l_{1}, l_{2}\right)\right)}{\operatorname{depth}\left(l_{1}\right)+\operatorname{depth}\left(l_{2}\right)}
$$

Using this modified notion of dot product, they compute the similarity between any two objects using the cosine measure.

In their second measure called the Optimistic Genealogy measure or OGM, they compute a "similarity contribution" for each element in one collection and then take the weighted average of these contributions to be the similarity between the two collections. The contribution of an element is determined by how good a "match" it has in the other collection. Let $T_{1}$ and $T_{2}$ be the induced trees for the collections $C_{1}$ and $C_{2}$ whose similarity we are trying to measure. For any element $l_{1}$ in $T_{1}$, they define $\mathrm{LCA}_{\mathrm{T} 1, \mathrm{~T} 2}\left(1_{1}\right)$ to be the ancestor of $1_{1}$ of greatest depth that is present in $\mathrm{T}_{2}$. They define:

$$
\operatorname{leafsim}_{T_{1}, T_{2}}\left(l_{1}\right)=\frac{\operatorname{depth}\left(L C A_{T_{1}, T_{2}}\left(l_{1}\right)\right)}{\operatorname{depth}\left(l_{1}\right)}
$$


The value of leafsim is the measure of how similar $1_{1}$ is to its best match in $T_{2}$. They then take the weighted average of individual leafsim values to compute the similarity between the two collections $\mathrm{C}_{1}$ and $\mathrm{C}_{2}$. They also discuss two other measures called the Balanced Genealogy Measure (BGM) and the Recursive Genealogy Measure (RGM) to deal with the cases where the same element occurs multiple times in an object. They evaluate these measures using a data set consisting of transcripts of undergraduate CS majors at Stanford University. They compare their approach against a traditional non-hierarchy aware measure called the Jaccard's Coefficient. They also perform a user study to understand how well the various measures matched human intuition. Their experiments proved that their measures that made use of the hierarchical information about the object gave better results than the traditional measures.

In [1], the authors make use of hierarchical organization of websites to compute the differences between two web sessions. They describe a method to compute the dissimilarity between two user Web sessions extracted from the Web access logs. They then use this dissimilarity measure to cluster the different user sessions using a fuzzy clustering algorithm. They derive their dissimilarity measure from two different similarity measures. In the first, they assume that all the URLs accessed in the sessions are totally distinct. The similarity between two sessions depends on the number of URLs they have in common. This is same as the cosine similarity measure. They denote this measure as $\mathbf{M}_{1 . \mathrm{kl}}$, where $\mathrm{k}$ and $\mathbf{l}$ are two user sessions. For their second similarity measure, they model a Website as a tree rooted at the server's 
document root. The different URLs form the nodes of the tree. They then make use of the overlap in the paths from the root of the tree to the corresponding nodes to measure the similarity between two URLs and thus between two user sessions. They denote this measure as $\mathbf{M}_{2 . \mathrm{kl}}$. They argue that each of these similarity measures have their merits and demerits. For the purpose of clustering, they define a dissimilarity measure as $d^{2}(k, l)=\left(1-M_{k l}\right)^{2}$, where, $M_{k l}$ is the maximum of $M_{1 . k l}$ and $M_{2 . k l}$. They make use of this dissimilarity measure to cluster different user session extracted for Web logs. For their experiments, they made use of the Web logs from the university Websites. They compare their approach with the publicly available apriori algorithm to generate association rules between the sessions. They found that their clustering algorithm that makes use of a graded notion of similarity produced larger coherent groups of user sessions than the other algorithm.

In [13], the authors present a very interesting algorithm called SimRank. Unlike some of the other algorithms that are based on the content within the objects or on the external information about the objects like their categories, they compute similarity between objects based on the structural context in which the objects occur within the domain. Their algorithm is based on the idea that "two objects are similar if they are related to similar objects." This in a way is a generalization of co-citation [9] where similarity of the citing documents is also considered, recursively. In their model, they represent the domain as a directed graph $\mathrm{G}=(\mathrm{V}, \mathrm{E})$ with objects as the nodes, $\mathrm{V}$ and the relationship between these objects as the edges, E. For example, these relationships can be the citations if we are looking at the scientific papers as the 
objects or they can be the hyperlinks in case of the Web documents. Their basic recursive equation for SimRank equation is defined as follows:

$$
s(a, b)=\frac{C}{|I(a)||I(b)|} \sum_{i=1}^{|I(a)||I(b)|} \sum_{j=1} s\left(I_{i}(a), I_{j}(b)\right)
$$

Where $s(a, b)$ is the similarity between objects $a$ and $b . s(a, b)=0$ if $a=b$. For any node belong to $\mathrm{V}, \mathrm{I}(\mathrm{v})$ represents the set of in-neighbors of $\mathrm{v}$. In the above equation, $\mathrm{C}$ is a constant between 0 and 1 . They consider $\mathrm{C}$ to be a decay factor. This is important because let's say an object $\mathrm{x}$ is related to $\mathrm{c}$ and $\mathrm{d}$. According to SimRank, $\mathrm{c}$ and $\mathrm{d}$ are similar to each other. In order to avoid concluding the $\mathrm{s}(\mathrm{c}, \mathrm{d})=\mathrm{s}(\mathrm{x}, \mathrm{x})=1$ ( since $s(x, x)=1)$, they use $s(c, d)=C . s(x, x)$. This, in a way is similar to the weight propagation factor that we use in our algorithm. For a graph of size $\mathrm{n}$, there will exist $\mathrm{n}^{2}$ SimRank equations, one for each pair of nodes. They find a solution to these equations by iterating to fixed-point. They represent $R_{k}(a, b)$ as the score between $\mathrm{a}$ and $\mathrm{b}$ at the iteration $\mathrm{k}$. They start with the base case where $\mathrm{R}_{0}(\mathrm{a}, \mathrm{b})=1$ if $\mathrm{a}$ $=\mathrm{b}$, and 0 if $\mathrm{a} !=\mathrm{b}$. They found that the scores stabilize within 5 iterations and hence they fix the number of iterations at 5. They also extensively discuss the computational overhead associated with their approach and also provide some pruning techniques that would reduce this overhead in case of some large applications.

For their experiments they used two data sets. Their first data set consisted of a collection of over 250,000 scientific research papers obtained from ResearchIndex 
(now CiteSeer[11]). Their second data set consisted of 1030 transcripts of undergraduate students. They compared their algorithm against the simple co-citation scheme. In their evaluation, they use a score, which they call the "actual score" generated by a coarse domain specific similarity measure. For scientific papers, they considered this to be "the fraction of q's citations also cited by p" where $\mathrm{p}$ and q are the papers whose similarity is being computed. They also considered another measure, "the fraction of words in q's title also in p's title". They measure the performance of an algorithm on object p using the difference between the algorithm's score and the "actual score". They found significant improvement by using their algorithm over the simple co-citation measures.

CiteSeer [4], which is a search engine for scientific publications in the field of computer and information sciences, employs a combination of algorithms to find related documents to the documents in its collection. They make use of a weighted combination of the TFIDF based algorithm, the LikeIt string edit distance algorithm and a citation similarity measure called CCIDF to come up with related documents. The TFIDF [22] based algorithm uses the normal cosine similarity between word vectors of the documents to find the matching documents. Using the LikeIt string edit distance measure, they try to match the headers of the documents to find related content. In the CCIDF measure, they make use of the citation information stored in their database to find related content. They consider two documents to be similar to each other if they share common citations. As in TFIDF, they weigh uncommon 
citations shared by two documents higher than the citations made by a large number of documents by using inverse weights.

\subsection{Tree Edit Distance}

The tree edit distance algorithm is a popular measure for tree comparison. The different variations and optimizations of this algorithm have been extensively researched in literature [5][14][15][19]. It was first introduced by Tai [14] in late 1970's as an extension to the string edit distance problem. The edit distance between two trees $\mathrm{A}$ and $\mathrm{B}$ is the minimum cost of transforming $\mathrm{A}$ into $\mathrm{B}$ by a sequence of elementary operations such as insertion, deletion and substitution of nodes. In it most generalized form the solution to the problem of finding the minimum cost of transformation was found to have quadratic complexity [5]. In the case of unordered trees the problem is NP-complete as discussed in [15]. Most of the solutions [14] were based on dynamic programming and were found to have a complexity of $\mathrm{O}\left(\mathrm{n}_{1} \mathrm{n}_{2} \mathrm{~h}_{1} \mathrm{~h}_{2}\right)$, where $\mathrm{n}_{1}$ and $\mathrm{n}_{2}$ are the sizes of the trees and $\mathrm{h}_{1}$ and $\mathrm{h}_{2}$ their heights. [7] is an excellent resource for algorithms pertaining to trees and graphs. It also provides some code implementation examples of these algorithms. We first present some formal definitions of a tree and an ordered tree taken from [7].

DEFINITION 1: A connected graph $G=(V, E)$ is said to be a tree if the underlying undirected graph has no cycles and there is a distinguished node $r \in V$, called the root of the tree and denoted by root[T] such that for all nodes $v \in V$, there is a path in $G$ from the root to node v. 
DEFINITION 2: An ordered tree is a tree in which relative order of the children is fixed for each node.

Several researchers have made use of modified and simplified versions of the tree edit distance algorithm to solve a variety of problems.

In [20] and [26], the authors make use of a modified version of this algorithm to detect similarities and differences between software programs. In [20], the authors use tree-matching algorithms to find similar java classes in software projects. This can help in efficient code reuse. They convert the abstract syntax tree (AST) representation of source code into trees using certain java tools. They then apply a tree edit distance algorithm on such trees to compute their similarity. In [26], the author presents an algorithm for identifying syntactic differences between two computer programs. He first uses a parser to convert the programs into parse trees. He then applies a generalized version of Sequence_Matching algorithm on these trees. The 'sequence matching' algorithm can be modeled as a tree-matching algorithm for trees of length 2 . The author applies this algorithm recursively at each level of the parse trees to find the syntactic differences between two computer programs.

Tree-matching algorithms have also been used to extract information from Websites [16][27]. In [16], the authors present a modified version of tree edit algorithm, which is used to extract news from Websites. Their algorithm called the restricted top down 
matching (RTDM), proceeds by finding all the identical sub trees that occur at the same level of the input trees. They first collect a set of Web documents, which is used as a training set. They cluster these documents and come up with a representative template tree for each of these clusters. They then match the other crawled documents from the Web to these trees to extract data from the corresponding sections. They make use of their RTDM algorithm for this matching. They tested their approach using a collection of documents from Brazilian news Websites and found that it works better than an existing top down edit distance algorithm.

In [27], the authors present their algorithm to extract Web data, which is based on partial tree alignment. Their extraction algorithm involves two steps. In the first step, they generate HTML tag trees for the Websites using the inherent hierarchical structure of HTML source code. In the second step, they align the tag trees generated in the first step using a tree-matching algorithm. The algorithm they use in this step is same as the one used in [26]. They first start with a seed tree denoted by $\mathrm{T}_{\mathrm{s}}$, which the tree with the maximum number of data fields. Then this seed tree is progressively grown, by matching it with the corresponding nodes in every other tree. If a match cannot be found for any node, the algorithm expands the seed tree by inserting this node into it. They evaluated this approach against their existing algorithm for data extraction and found that this new algorithm based on tree alignment gave better results. 
XML documents are good candidates for applications of tree algorithms because of their hierarchical structure. In [2][28][29], different variations of tree edit distance algorithm have been applied to find similarities between xml documents. In [2], the authors apply a modified version of the tree edit distance algorithm to find structural similarity between two XML documents. Their goal was to cluster a collection of XML documents using their similarity measure. This allows them to apply other algorithms on these clusters to come of with a DTD for each of them. They represent each XML document as a labeled ordered tree with a node corresponding to each element and to each attribute. XML documents can have repeating elements and so two documents produced from the same DTD can have very different sizes. They note that because of this nature of XML documents, any edit distance metric that permits changes to only one node at a time will find large distances between such a pair of documents, and consequently will not recognize that these documents should be clustered together as being derived from the same DTD. In their edit distance metric they define certain allowable operations where they can insert or delete an entire sub-tree from an XML document. They evaluate their algorithm by using it to cluster a set of XML documents whose DTDs are previously know. They then measure how closely the documents in the clusters match to their corresponding DTDs.

Many applications that deal with XML documents require them to conform to a given DTD. In [28], the author presents an algorithm to find an edit script that optimally transforms an invalid XML document into a valid one w.r.t. a given DTD. He models 
the XML document and the DTD as trees and applies a tree edit distance algorithm to come up with the edit script. In [29] also, the authors solve a similar problem of matching an XML document and a DTD using a tree edit distance algorithm. The represent XML documents as trees and the schema or DTD as a normalized regular hedge grammar (NRHG). They use their matching algorithm to classify XML documents. They tested their classification system on the data from XML Mining challenge and compared it with a naïve Bayesian classifier.

It can be seen from the above discussion that the concept of tree matching can be used in a variety of applications. The matching algorithm can be modified and simplified to suit the requirements of the applications. In our case, we deal with concept trees derived from a common parent taxonomy. This allows us to use a simplified version of the tree edit distance algorithm. In the next chapter we will discuss our idea of modeling documents as concept trees and present an algorithm to match these concept trees. 


\section{Chapter 3 - Approach}

\subsection{Overview}

The matching algorithm we use is the Tree Edit Distance algorithm. The algorithm finds the cost of transforming one tree to the other. This involves a series of operations on one of the trees which include insertion, deletion or substitution of nodes. Each of these operations has a cost associated with it and the lower the total cost of transformation between two trees the more similar they are. All of our concept trees are part of a common parent tree and each node of the tree has a label and a weight associated with it. The label is the category ID or the category name and the weight is the weight of the corresponding category for that document obtained from the classifier. Hence, we consider these trees as rooted ordered labeled weighted trees. The process of building these trees from a vector of concepts and associated weights is discussed in detail in Section 3.2.2. The overall system consists of three modules namely, the Classification module, the Tree Builder module and the Tree Matching module. The interaction between these three modules is shown in the following figure and each module is clearly discussed in the sections that follow it. 


\subsection{System Architecture}

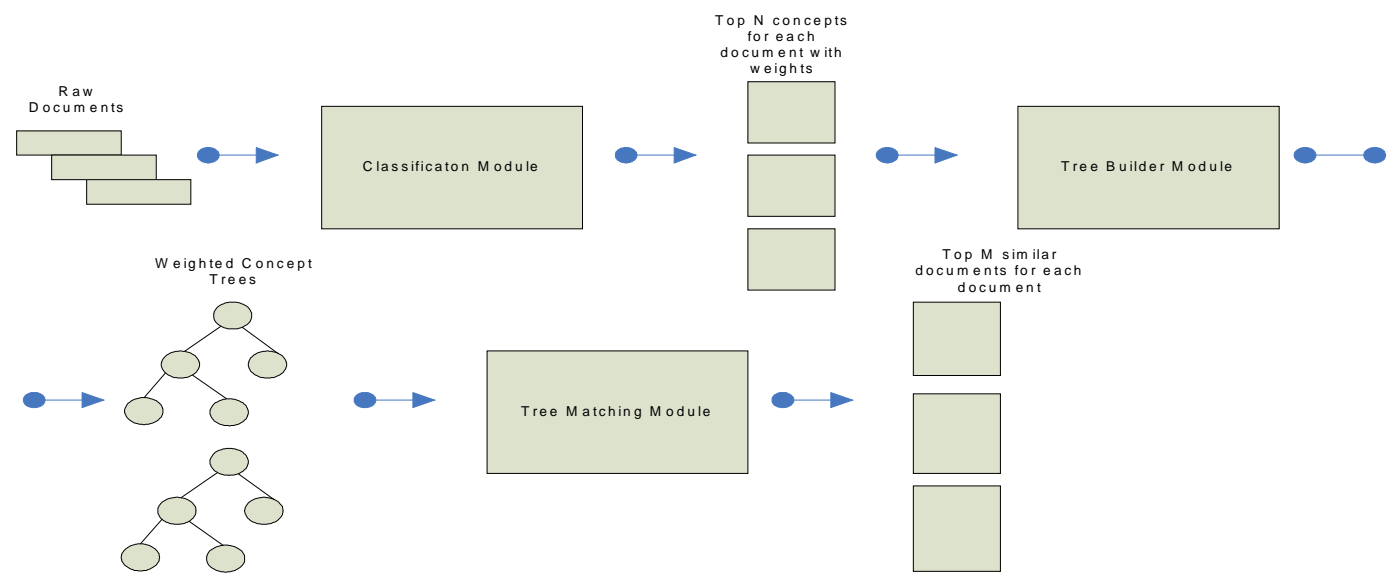

Figure 1: System Architecture

\subsubsection{Classification Module}

The first step in our approach is to classify the set of input documents. Any classifier can be used for this purpose. The process of classification normally starts by collecting a set of training documents and choosing the taxonomy of categories relevant to the domain that the input documents belong to. The training set contains documents which are pre-assigned one or more categories in the taxonomy either manually or by some other method. Classification consists of two stages, the training stage and the classification stage. In the training stage, the classifier uses the training documents to build a model for each category in the taxonomy. This model will be used to classify the input documents at the classification stage. The output of the classification stage is a list of top categories for each input document along with their weights. There are a number of classifiers available and the choice of the classifier depends on a lot of different factors. Some of these are the domain of the input 
documents, the size of these documents, the amount of training data available, and the size of the taxonomy being used. It is always better to conduct a few experiments before choosing any classifier. We conducted some experiments before choosing Rocchio as the classifier for our dataset and we discuss the results of these experiments in Section 4.1.

\subsubsection{Tree Builder Module}

This module generates the weighted concept tree for each input document using their concept vectors obtained from the classification stage. The tree is generated by recursively finding the parent of the current node until we reach the root node, for each category in the concept vector. Weights are assigned to parents according to the following equation:

$$
\mathrm{W}_{\mathrm{P}}=\alpha * \mathrm{~W}_{\mathrm{C}}
$$

Where, $\mathrm{W}_{\mathrm{P}}$ is the weight of the parent.

$\mathrm{W}_{\mathrm{C}}$ is the weight of the child. and

' $\alpha$ ' is a weight propagation factor.

The weight propagation factor is used to determine how much of the child's weight can be assigned to its parent. In other words, it is used to determine how closely a document belongs to a parent category given that it belongs to a child category. When $\alpha=0$, the parents will not be assigned any part of the child's weight. In this case, we would essentially be dealing with concept vectors themselves, as the parents will not have any role in the matching process. This will be clear when we discuss the 
matching algorithm in the next Section. The pseudo code for the tree builder function is shown in the figure below.

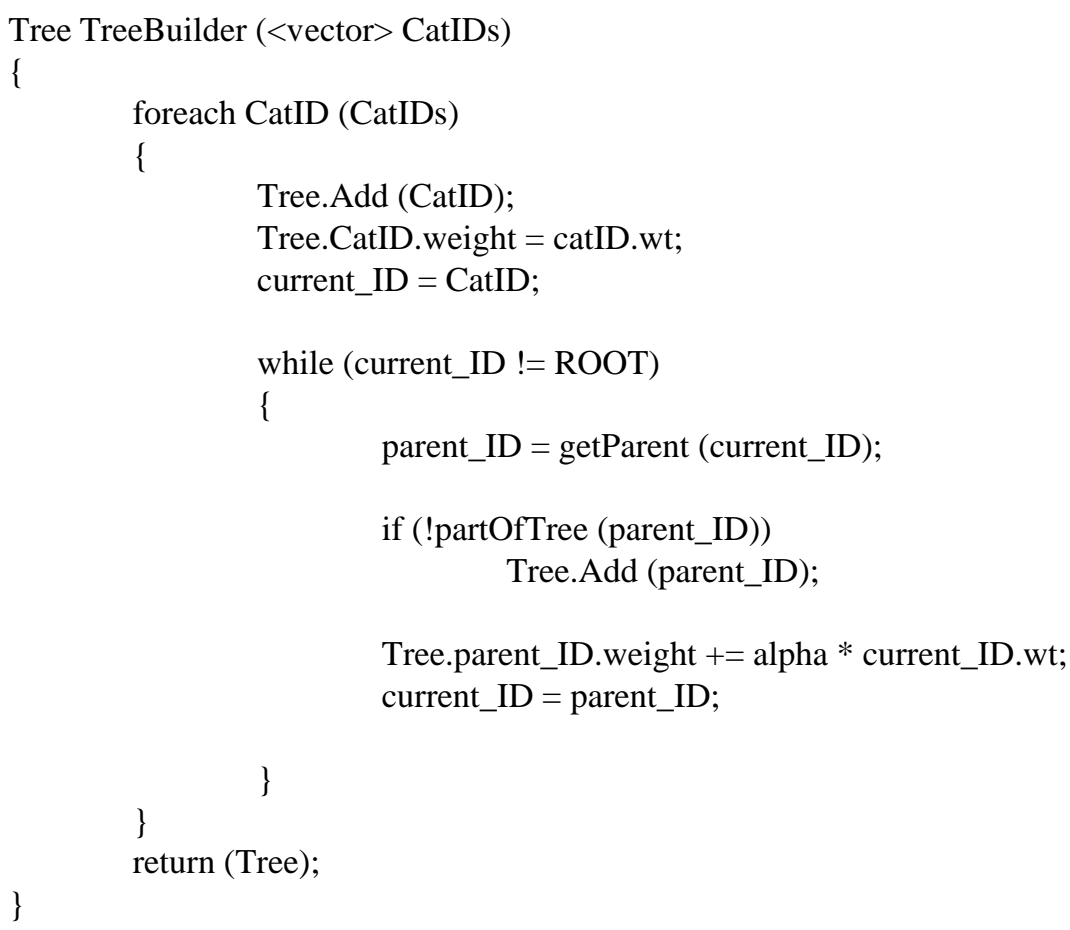

Figure 2: Pseudo code for Tree Builder

Since all the concepts are part of the same taxonomy, all the trees generated have the same root and the concepts at each level and their ordering also remains the same. In order to better understand the process of generating the trees, consider the sample taxonomy shown in the figure below. It is three levels deep and each node represents a unique concept. 


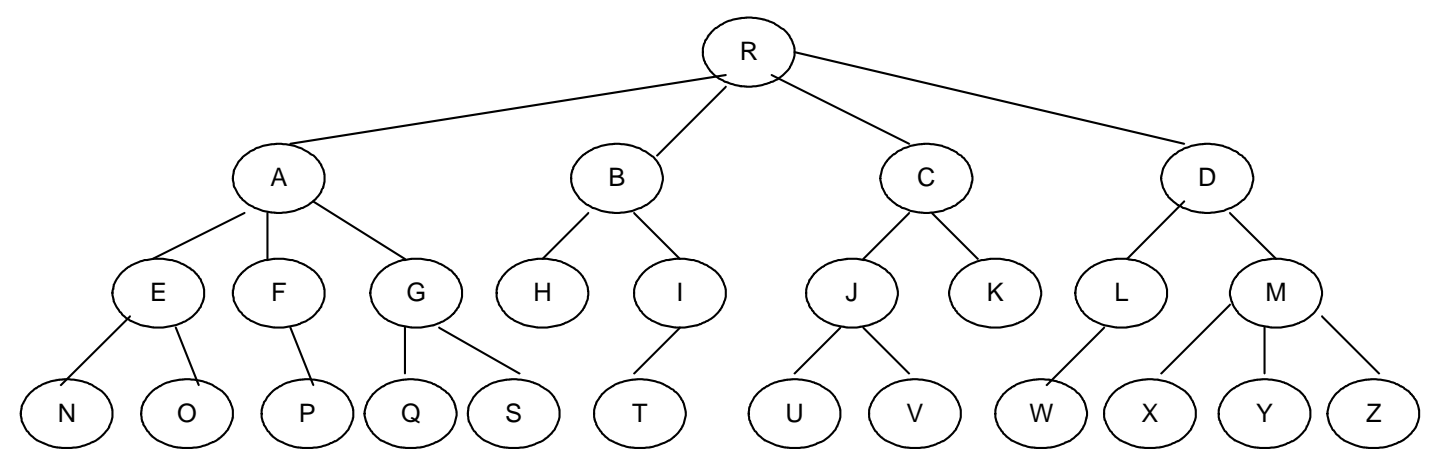

Figure 3: Sample Taxonomy

Let the concept vectors obtained from the classification stage for two sample documents D1 and D2 be the following.

$$
\begin{aligned}
& \mathrm{D} 1=\{(\mathrm{F}, 40),(\mathrm{C}, 30),(\mathrm{E}, 20),(\mathrm{A}, 10)\} \\
& \mathrm{D} 2=\{(\mathrm{U}, 60),(\mathrm{V}, 40),(\mathrm{A}, 20),(\mathrm{K}, 10)\}
\end{aligned}
$$

In the above vectors the ordered pair (F, 40), represents the concept ID and its weight and the same applies for all the other ordered pairs. The step-by-step process of transforming the concept vector for document $\mathrm{D} 2$ to a concept tree $\mathrm{T} 2$ using the algorithm listed in figure 2 and the taxonomy in figure 3 , is shown in the following figure. The weight propagation factor or ' $\alpha$ ' used here is 0.5 . We can clearly see how the weights get propagated from the child to the parent in this figure.

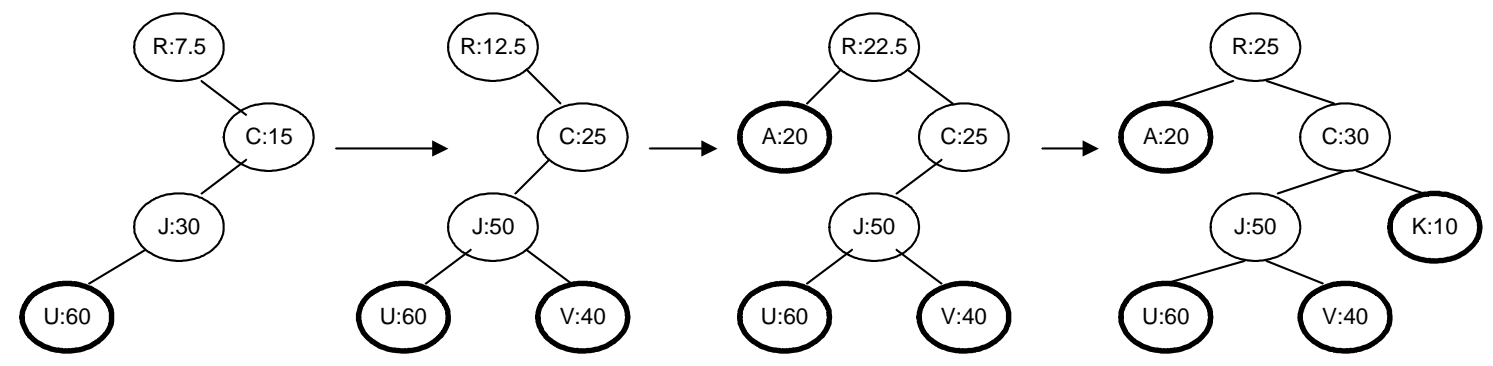


Figure 4: Step by step process of building the concept tree T2 of Document D2

The concept tree for D1 is obtained in a similar fashion and it is shown below.

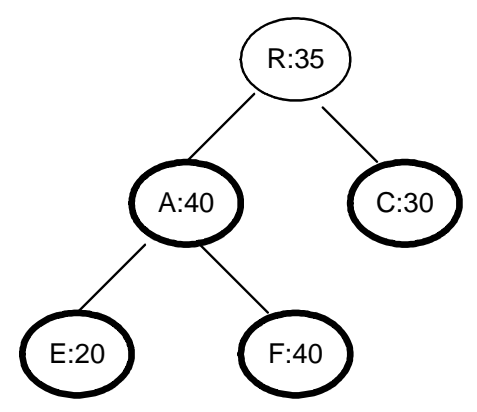

Figure 5: The concept tree T1 for the document D1

In the above figures, the nodes that contain concepts that are actually present in the concept vectors (i.e. those that are not added as part of the tree building process) are shown in bold. Each node is labeled with the concept ID and also its weight. In figure 5 , it can be seen how category A which had an initial weight of 10 , finally has a weight of 40 after the concept tree for D1 is generated. The additional weight is because it is the parent of two other nodes. In this way, each input document is transformed into a concept tree in the Tree Builder module. A tree-matching algorithm such as tree edit distance can be applied to a pair of trees generated in this fashion. The matching module is used for this purpose and it is discussed in the next section. 


\subsubsection{Matching Module}

The similarity between two concept trees is computed by using a tree-matching algorithm called the tree edit distance. The three operations we consider for transforming one tree to the other are insertion, deletion and substitution of nodes. Since our concept trees have weighted nodes, the cost associated with insertion or deletion of a node is equal to the corresponding weight on the node that is being inserted or deleted. Similarly, the cost associated with substitution of a node with a node from the other tree is the difference between their corresponding weights. Since all our concept trees are part of the same taxonomy; we can assume that the nodes which are not actually present in a given concept tree to be virtually present in it with a weight of zero. Then we would have the same complete concept tree for each document and the process of transforming one tree to the other would come down to a series of substitution operations. Either way, the goal is to find the least cost of transformation between two trees. The lower the cost, the more similar they are.

In order to better understand our approach, we show the steps involved in the transformation between the sample trees built in Section 3.2.2 in the following figure. In the figure, at each step of the transformation, we show the operation being performed and also the total cost after that operation (i.e. the previous cost + the cost of the operation). The insertion of a node $\mathrm{X}$ is denoted by $\operatorname{Add}(\mathrm{X})$, the deletion by $\operatorname{Del}(\mathrm{X})$ and the substitution by $\operatorname{Sub}(\mathrm{X})$. 
The initial value of cost, $\mathrm{C}$, is zero.

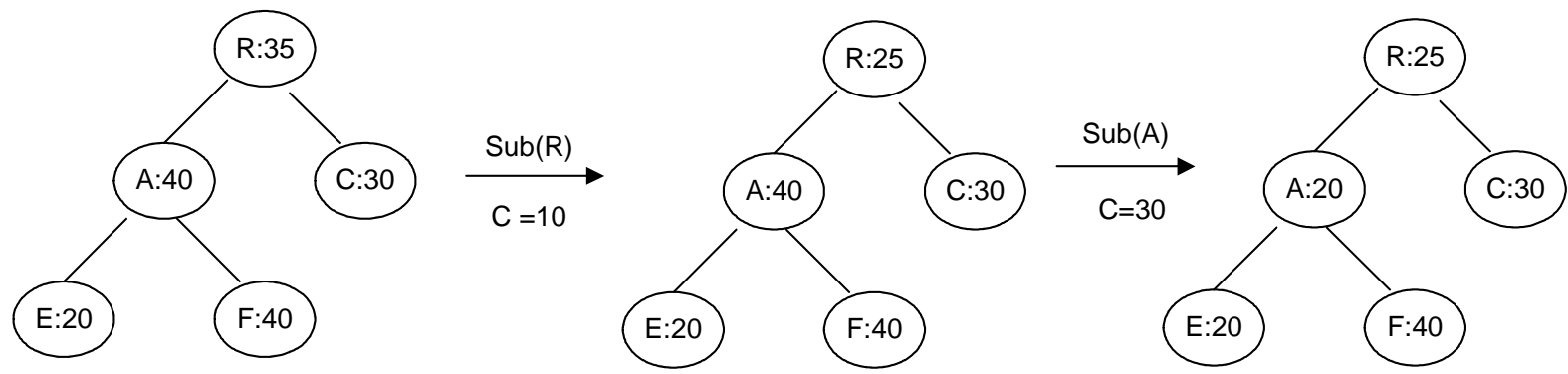

T1
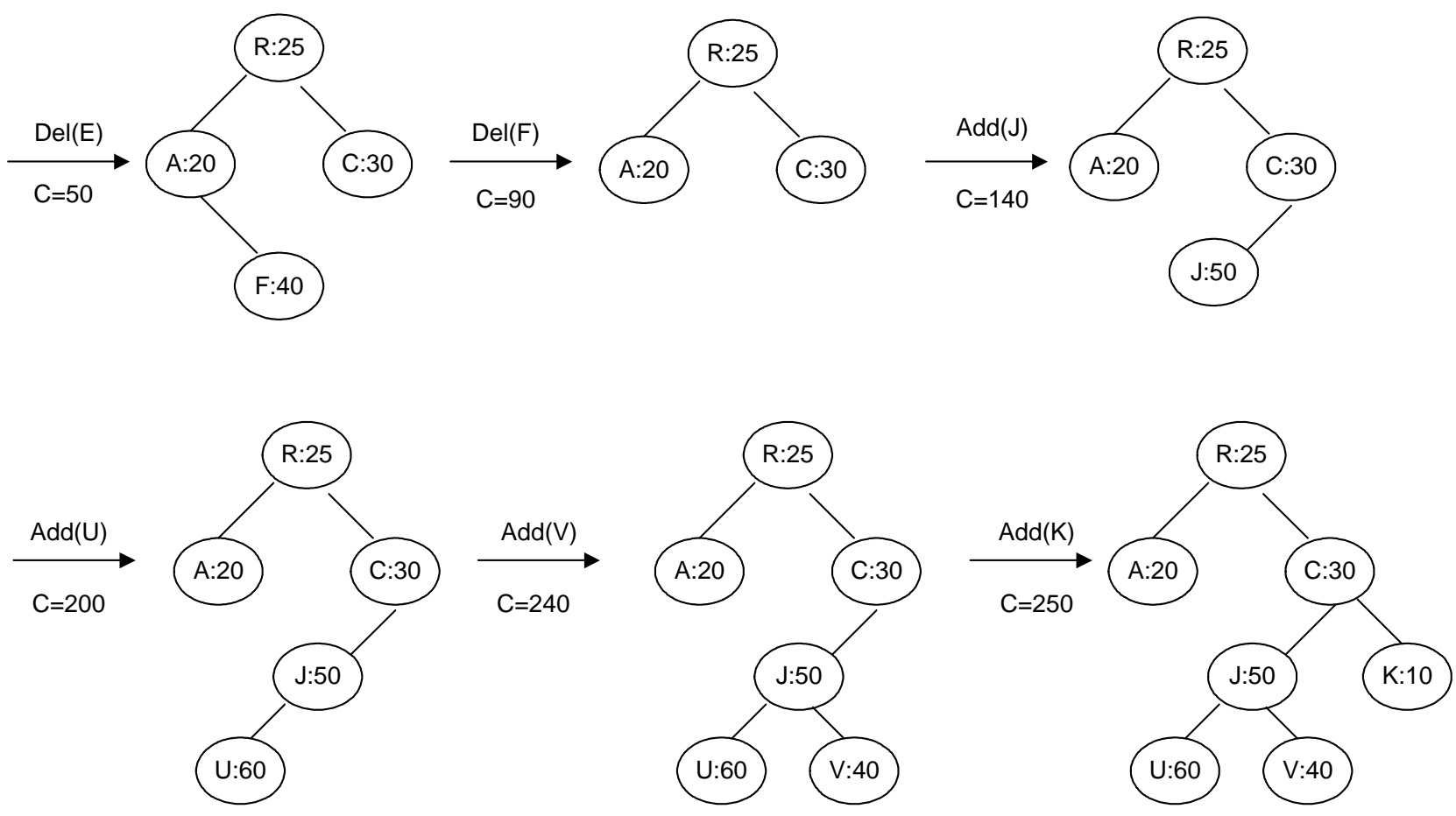

T2

Figure 6: The step-by-step process of transforming concept tree T1 to concept tree T2 
The pseudo code for our tree edit distance algorithm is shown in the following figure.

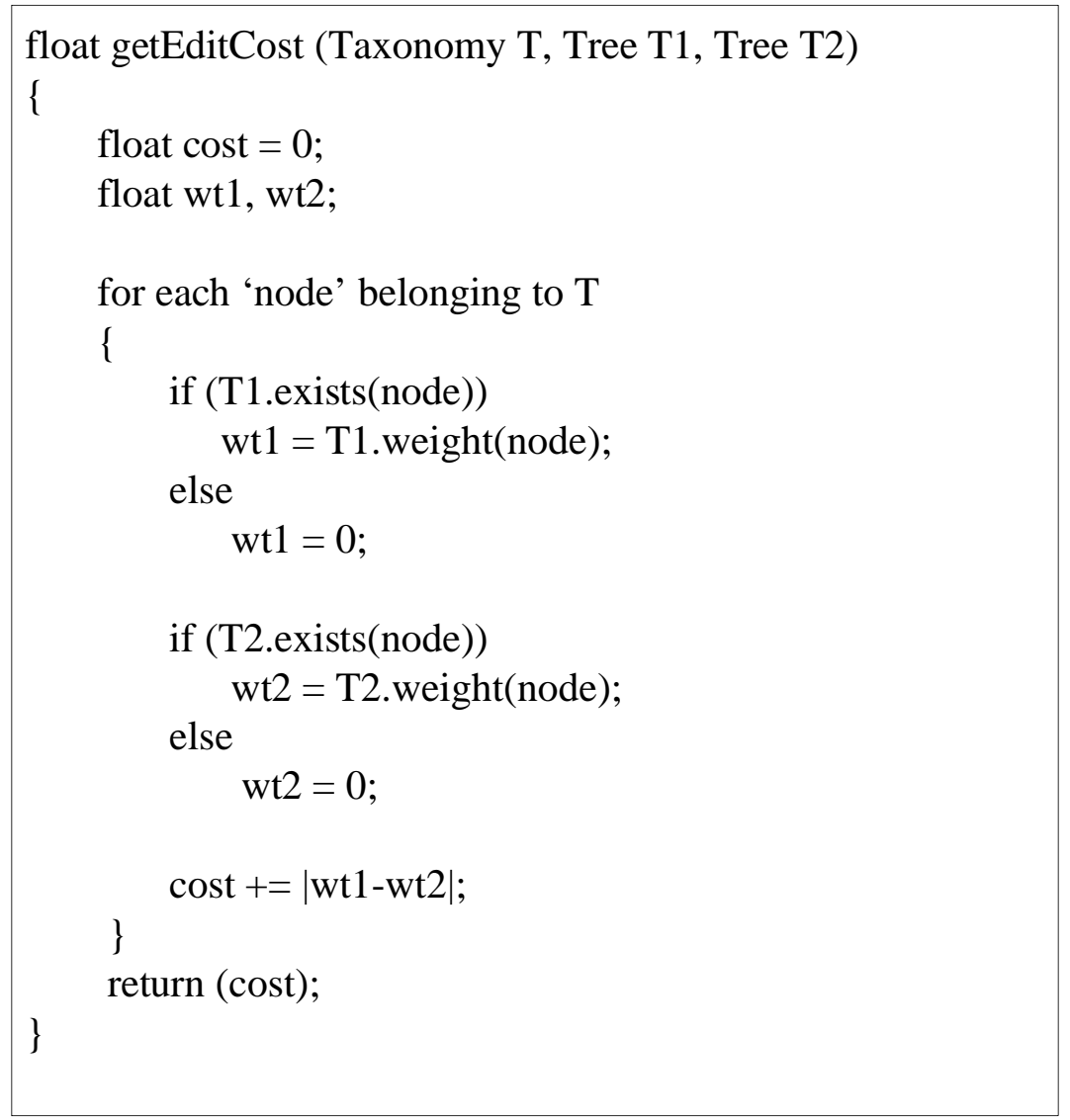

Figure 7: Pseudo code for the edit distance algorithm

The complexity of our algorithm is $\mathrm{O}(\mathrm{N})$, where $\mathrm{N}$ is the total number of nodes or concepts in the taxonomy. The reason for such a simple algorithm is because of the fact that all our concept trees are derived using the same base tree or taxonomy. The nodes or concepts at each level are unique and their order is also the same. Much more complex algorithms would be necessary if the trees belonged to different taxonomies where the same concepts exist in different levels with non-uniform ordering. Since these cases are not pertinent to our current work, we do not consider them further. 


\section{Chapter 4 - Evaluation Methodology and Results}

\subsection{Dataset and Methodology}

We used a subset of the CiteSeer [11] collection for our evaluation. CiteSeer is an automated digital library for scholarly literature in computer and information sciences. It consists of over 700,000 academic science papers and over 10 million citations. We randomly selected 50,100 documents from this collection to serve as our dataset. In order to build concept trees for each of these documents, we first had to classify them. We used the ACM's classification hierarchy called the CCS or Computing Classification System [10] as our taxonomy because it covers all of the topics for the documents in the citeseer collection. This taxonomy is of three levels deep with a total of 368 categories.

We conducted a series of experiments to select the best classifier for our purpose. Our training data consisted of documents from the citeseer collection that were manually classified by their authors into one or more of the ACM categories. We experimented with Rocchio, SVM and Knn classifiers. The results of these experiments are shown in the tables below.

\begin{tabular}{|l|r|r|r|r|r|r|r||}
\hline \# Training Docs/Category & $\mathbf{3}$ & $\mathbf{5}$ & $\mathbf{7}$ & $\mathbf{9}$ & $\mathbf{1 1}$ & $\mathbf{1 3}$ & $\mathbf{1 5}$ \\
\hline \# Categories & 223 & 204 & 183 & 171 & 155 & 146 & 136 \\
\hline & & & & & & & \\
\hline Rocchio & 66.10 & 75.09 & 79.51 & 80.79 & 82.93 & $\mathbf{8 6 . 9 1}$ & 85.96 \\
\hline SVM & 5.76 & 5.58 & 6.24 & 8.76 & 9.35 & 10.47 & 26.4 \\
\hline Knn & 62.71 & 71.75 & 76.64 & 78.17 & 80.49 & 83.25 & 83.15 \\
\hline
\end{tabular}

Table 1: Experiment 1 - Using all words for training and top 50 for testing 


\begin{tabular}{||l|r|r|r|r|r|r|r||}
\hline \# Training Docs/Category & $\mathbf{3}$ & $\mathbf{5}$ & $\mathbf{7}$ & $\mathbf{9}$ & $\mathbf{1 1}$ & $\mathbf{1 3}$ & $\mathbf{1 5}$ \\
\hline \# Categories & 223 & 204 & 183 & 171 & 155 & 146 & 136 \\
\hline & & & & & & & \\
\hline Rocchio & 65.09 & 73.61 & 79.51 & 81.22 & 80.49 & 86.39 & 85.39 \\
\hline SVM & 29.15 & 38.48 & 44.67 & 56.4 & 58.32 & 67.47 & 69.12 \\
\hline Knn & 63.05 & 72.12 & 77.05 & 79.04 & 80 & 81.68 & 82.02 \\
\hline
\end{tabular}

Table 2: Experiment 2 - Using top 200 words for training and top 50 for testing

\begin{tabular}{||l|r|r|r|r|r|r|r||}
\hline \# Training Docs/Category & $\mathbf{3}$ & $\mathbf{5}$ & $\mathbf{7}$ & $\mathbf{9}$ & $\mathbf{1 1}$ & $\mathbf{1 3}$ & $\mathbf{1 5}$ \\
\hline \# Categories & 223 & 204 & 183 & 171 & 155 & 146 & 136 \\
\hline & & & & & & & \\
\hline Rocchio & 67.12 & 74.72 & 80.33 & 82.97 & 83.42 & 85.86 & 84.83 \\
\hline SVM & 48.81 & 64.68 & 67.05 & 68.45 & 74.67 & 77.49 & 79.78 \\
\hline Knn & 65.09 & 72.12 & 78.69 & 81.22 & 80.98 & 83.25 & 84.27 \\
\hline
\end{tabular}

Table 3: Experiment 3 - Using all words for training and all words for testing

It can be seen that Rocchio performed better than the other two classifiers at all training levels in all the three experiments. The best performance was obtained when we used 13 training documents per category while using all the words (excluding stop words) in the document for training and the top 50 words in the document for testing. There were a total of 146 categories with at least 13 training documents per category and we used these categories for classifying our dataset.

Once we decided on using the Rocchio classifier, we categorized all the input documents and obtained the top 20 categories for each document and built the concept trees for these documents using the algorithm discussed in Section 3.2.2. While building these concept trees, we tried five different weight propagation factors or ' $\alpha$ '. See Section 3.2.2 for a discussion on the weight propagation factor. One of our 
goals is to find the best value for ' $\alpha$ '. The ' $\alpha$ ' values that were tried are $1,0.75,0.5$, 0.25 and 0 . We discuss the results obtained with each of these ' $\alpha$ 's in Section 4.3.

It is always difficult to evaluate the kind of work presented in this thesis. This is because of the lack of pre-defined "truth" for the relatedness between documents. Most of the researchers utilize user studies in such cases. In the same way, to evaluate the effectiveness of our approach to compute document similarity, we performed a user study. We randomly selected 14 documents from our dataset to serve as the query documents. For each of these query documents, we computed the top 10 most similar documents from amongst the remaining dataset using our 'concept tree' matching algorithm. We also computed the top 10 most similar document using the baseline algorithm to be discussed in Section 4.2.

We used nine volunteers for our user study. All of these were graduate students in EECS department at the University of Kansas. Each of these volunteers rated a set of potentially related documents to no more than five query documents. These potentially related documents consisted of those obtained using both our algorithm and also the baseline algorithm. We presented them in random order to the users in order to avoid any bias. The users were then asked to score the documents for relatedness as 0 (not related), 1 (somewhat related) or 2(very much related). At the end of the user study, we had three ratings for each of the potentially related documents to each of the query documents. 
When calculating precision in top 10 and top 3, we considered three different definitions of a good recommendation or "hit". These are defined below.

1. At least 2 of 3: A document is considered to be a good recommendation if at least two of the three users find it related.

2. At least 1 of 3: A document is considered to be a good recommendation if at least one of the three users find it related.

3. Average Score $>=1$ : A document is considered to be a good recommendation if the average user rating or score for that document is greater than or equal to 1.

According to us, the third definition of a hit above is very strong, the first is reasonable and the second is a bit weak. In the next section, we discuss our baseline algorithm and in the sections that follow it, we present the results of our user study.

\subsection{Overview of the Baseline}

Our baseline algorithm is based on the vector space model wherein each document is represented as a vector of words and their TFIDF weights. The similarity between two documents is computed by calculating the dot product between their word vectors. The current text based recommender system in CiteSeer [11] makes use of this approach. In order to build the baseline for our evaluation, we first indexed all the documents in our dataset except those being used as test documents. We used the Keyconcept [23] indexer for this purpose. By treating the top 50 words based on 
TFIDF score for each of the test documents as a query against the index, we retrieved the top 10 results in each case. These were combined with the other top 10 matches obtained using our new 'concept tree' matching algorithm for each value of ' $\alpha$ ' and presented to the users for evaluation. The comparison of the results for the baseline with those for our algorithm is presented in the next section.

\subsection{Results of the user study for our system}

In the following sections, we report the results from our user study and discuss them. In the tables below, we denote the baseline as M0. As we have noted earlier, we tried five different weight propagation factors or ' $\alpha$ ' for our algorithm during our experiments in order to find out the best performing ' $\alpha$ '. We denote each of these different cases as M1, M2, M3, M4 and M5. In M5, we used a weight propagation factor of 0 . As we have discussed earlier, in this case, the parents would get zero weight from their children and we would essentially be using the concept vectors themselves in the matching process. In our analysis of the results we will see how this case performed against the other cases that actually took the parent-child relationships between the concepts into account. For each of the three definitions of a "hit" discussed in Section 4.1, we calculate the precision in Top-10 and also in Top-3. Finding out the precision in top three results is important because in case of document recommendations, users are generally interested only in the top two or three recommendations. 


\subsubsection{Results for 'At least 2 of 3' measure}

The table below shows the precision in top ten results for each query in case of all the six methods.

\begin{tabular}{|r|r|r|r|r|r|r|}
\hline Query\# & \multicolumn{1}{l|}{ M0 } & M1 ( $\mathbf{\alpha = 1})$ & $\mathbf{M 2}(\boldsymbol{\alpha}=\mathbf{0 . 7 5})$ & $\mathbf{M 3}(\boldsymbol{\alpha}=\mathbf{0 . 5})$ & $\mathbf{M 4}(\boldsymbol{\alpha}=\mathbf{0 . 2 5})$ & $\mathbf{M 5}(\boldsymbol{\alpha}=\mathbf{0})$ \\
\hline & & & & & & \\
\hline $\mathbf{1}$ & 50 & 60 & 60 & 60 & 50 & 40 \\
\hline $\mathbf{2}$ & 40 & 80 & 70 & 70 & 60 & 60 \\
\hline $\mathbf{3}$ & 70 & 80 & 70 & 80 & 80 & 90 \\
\hline $\mathbf{4}$ & 10 & 30 & 30 & 40 & 40 & 40 \\
\hline $\mathbf{5}$ & 70 & 70 & 80 & 80 & 70 & 80 \\
\hline $\mathbf{6}$ & 50 & 30 & 30 & 30 & 40 & 50 \\
\hline $\mathbf{7}$ & 90 & 30 & 50 & 60 & 70 & 60 \\
\hline $\mathbf{8}$ & 30 & 70 & 70 & 70 & 70 & 70 \\
\hline $\mathbf{9}$ & 20 & 40 & 40 & 40 & 50 & 40 \\
\hline $\mathbf{1 0}$ & 20 & 40 & 40 & 40 & 50 & 70 \\
\hline $\mathbf{1 1}$ & 70 & 90 & 100 & 90 & 90 & 90 \\
\hline $\mathbf{1 2}$ & 20 & 20 & 20 & 20 & 40 & 30 \\
\hline $\mathbf{1 3}$ & 20 & 60 & 60 & 60 & 60 & 40 \\
\hline $\mathbf{1 4}$ & 100 & 80 & 90 & 80 & 90 & 90 \\
\hline & & & & & & \\
\hline Overall & 47.14 & 55.71 & 57.86 & 58.57 & 61.43 & 60.71 \\
\hline
\end{tabular}

Table 4: Precision in Top-10 for definition-1 of a "hit"

It can be seen from the above table that on the whole, our algorithm has performed better than the baseline for all the values of ' $\alpha$ '. Our algorithm gave the best overall performance in the case when ' $\alpha$ ' $=0.25$ (M4). M4 also performed better than M5, where we just use the concept vectors in the matching process. It can also be seen that for 11 out of 14 queries, our algorithm M4 has performed better or at least as well as the baseline.

In the table below, we look at the results for precision in top three for each query in all the six methods.

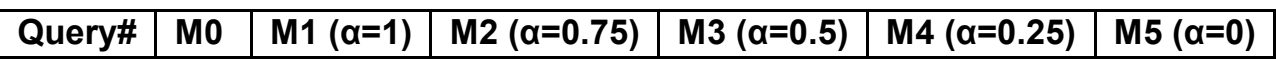




\begin{tabular}{|r|r|r|r|r|r|r|}
\hline & & & & & & \\
\hline $\mathbf{1}$ & 67 & 67 & 100 & 67 & 67 & 67 \\
\hline $\mathbf{2}$ & 0 & 67 & 100 & 100 & 100 & 33 \\
\hline $\mathbf{3}$ & 100 & 100 & 100 & 100 & 100 & 100 \\
\hline $\mathbf{4}$ & 0 & 67 & 33 & 33 & 100 & 100 \\
\hline $\mathbf{5}$ & 100 & 67 & 67 & 67 & 67 & 67 \\
\hline $\mathbf{6}$ & 67 & 67 & 67 & 67 & 67 & 100 \\
\hline $\mathbf{7}$ & 100 & 33 & 67 & 33 & 67 & 67 \\
\hline $\mathbf{8}$ & 0 & 100 & 100 & 100 & 100 & 67 \\
\hline $\mathbf{9}$ & 33 & 33 & 67 & 67 & 67 & 100 \\
\hline $\mathbf{1 0}$ & 33 & 67 & 67 & 67 & 33 & 33 \\
\hline $\mathbf{1 1}$ & 100 & 100 & 100 & 100 & 100 & 100 \\
\hline $\mathbf{1 2}$ & 0 & 0 & 0 & 33 & 33 & 33 \\
\hline $\mathbf{1 3}$ & 0 & 33 & 33 & 33 & 0 & 0 \\
\hline $\mathbf{1 4}$ & 100 & 100 & 100 & 100 & 100 & 100 \\
\hline & & & & & & 61.5 \\
\hline Overall & 50 & 64.36 & 71.5 & 69.07 & 71 & 69.07 \\
\hline
\end{tabular}

Table 5: Precision in Top-3 for definition-1 of a "hit"

As can be seen from the table above, even in precision at top three results, our algorithm has performed better than the baseline for all the ' $\alpha$ ' values. The best overall performance was obtained for ' $\alpha$ ' $=0.25$ (M4). For 12 out of 14 queries, M4 has performed at least as well as or better than the baseline. It can also be seen that 3 out of 4 cases that actually took the parent-child relationships into account performed better than M5, where we were using just the concept vectors.

\subsubsection{Results for 'At least 1 of 3 ' measure}

The table below shows the results for the precision in top ten when we consider the second definition of a "hit" as discussed in Section 4.1.

\begin{tabular}{|c|c|c|c|c|c|c|}
\hline Query\# & MO & M1 $(\alpha=1)$ & M2 ( $\alpha=0.75)$ & M3 $(\alpha=0.5)$ & M4 ( $\alpha=0.25)$ & M5 $(\alpha=0)$ \\
\hline 1 & 90 & 80 & 80 & 80 & 80 & 80 \\
\hline 2 & 50 & 100 & 100 & 90 & 90 & 90 \\
\hline 3 & 80 & 100 & 100 & 100 & 100 & 100 \\
\hline 4 & 90 & 80 & 80 & 80 & 80 & 80 \\
\hline 5 & 100 & 90 & 100 & 100 & 100 & 100 \\
\hline 6 & 60 & 80 & 80 & 90 & 100 & 100 \\
\hline
\end{tabular}




\begin{tabular}{|r|r|r|r|r|r|r|}
\hline $\mathbf{7}$ & 100 & 90 & 90 & 100 & 100 & 100 \\
\hline $\mathbf{8}$ & 80 & 100 & 100 & 90 & 90 & 90 \\
\hline $\mathbf{9}$ & 60 & 70 & 70 & 70 & 70 & 70 \\
\hline $\mathbf{1 0}$ & 100 & 100 & 100 & 100 & 100 & 100 \\
\hline $\mathbf{1 1}$ & 100 & 90 & 100 & 100 & 100 & 100 \\
\hline $\mathbf{1 2}$ & 20 & 50 & 50 & 50 & 70 & 70 \\
\hline $\mathbf{1 3}$ & 60 & 90 & 90 & 90 & 90 & 80 \\
\hline $\mathbf{1 4}$ & 100 & 100 & 100 & 90 & 90 & 90 \\
\hline & & & & & & \\
\hline Overall & 77.86 & 87.14 & 88.57 & 87.86 & 90 & 89.29 \\
\hline
\end{tabular}

Table 6: Precision in Top-10 for definition-2 of a "hit"

Our algorithm performed better than the baseline for all the values of ' $\alpha$ '. The best overall performance was obtained for M4 when ' $\alpha$ ' $=0.25$. For 11 out of 14 queries, M4 performed better than or at least as good as the baseline.

In the table below, we look at the precision in top three results for each query in all the six methods.

\begin{tabular}{|c|c|c|c|c|c|c|}
\hline Query\# & MO & M1 ( $\alpha=1)$ & M2 $(\alpha=0.75)$ & M3 $(\alpha=0.5)$ & M4 ( $\alpha=0.25)$ & M5 $(\alpha=0)$ \\
\hline & & & & & & \\
\hline 1 & 100 & 66 & 100 & 100 & 100 & 100 \\
\hline 2 & $\overline{0}$ & 100 & 100 & 100 & 100 & 66 \\
\hline 3 & 100 & 100 & 100 & 100 & 100 & 100 \\
\hline 4 & 66 & 100 & 100 & 100 & 100 & 100 \\
\hline 5 & 100 & 100 & 100 & 100 & 100 & 100 \\
\hline 6 & 66 & 100 & 100 & 100 & 100 & 100 \\
\hline 7 & 100 & 66 & 100 & 100 & 100 & 100 \\
\hline 8 & 66 & 100 & 100 & 100 & 100 & 66 \\
\hline 9 & 66 & 66 & 66 & 66 & 66 & 100 \\
\hline 10 & 100 & 100 & 100 & 100 & 100 & 100 \\
\hline 11 & 100 & 100 & 100 & 100 & 100 & 100 \\
\hline 12 & 0 & 33 & 33 & 66 & 66 & 66 \\
\hline 13 & 33 & 66 & 66 & 66 & 66 & 66 \\
\hline 14 & 100 & 100 & 100 & 100 & 100 & 100 \\
\hline Overall & 71.21 & 85.5 & 90.36 & 92.71 & 92.71 & 90.29 \\
\hline
\end{tabular}

Table 7: Precision in Top-3 for definition-2 of a "hit" 
It can be seen from the results that our algorithm again performed better than the baseline for all the values of ' $\alpha$ '. The best overall performance was obtained in the cases M3 and M4 where ' $\alpha$ ' is equal to 0.5 and 0.25 respectively. 3 out of 4 cases that took the concept hierarchy into account performed better than the concept vector method M5. It can also be seen that for all the 14 queries, M4 has done better than or at least as good as the baseline.

\subsubsection{Results for 'Average score $>=1$ ' measure}

The table below shows the precision in top ten results for all the queries in case of all the six methods. We used the third definition of a "hit" discussed in Section 4.1 for these results.

\begin{tabular}{|c|c|c|c|c|c|c|}
\hline Query\# & MO & M1 $(\alpha=1)$ & M2 ( $\alpha=0.75)$ & M3 (a=0.5) & M4 ( $\alpha=0.25)$ & M5 $(\alpha=0)$ \\
\hline & & & & & & \\
\hline 1 & 30 & 30 & 30 & 30 & 20 & 20 \\
\hline 2 & 20 & 40 & 40 & 50 & 50 & 50 \\
\hline 3 & 70 & 50 & 50 & 60 & 60 & 50 \\
\hline 4 & 10 & 20 & 10 & 20 & 20 & 20 \\
\hline 5 & 50 & 30 & 40 & 50 & 50 & 50 \\
\hline 6 & 50 & 20 & 20 & 20 & 20 & 20 \\
\hline 7 & 50 & 20 & 20 & 20 & 30 & 30 \\
\hline 8 & 30 & 50 & 50 & 40 & 40 & 40 \\
\hline 9 & 10 & 20 & 20 & 20 & 30 & 30 \\
\hline 10 & 10 & 30 & 30 & 30 & 40 & 40 \\
\hline 11 & 50 & 60 & 60 & 70 & 70 & 60 \\
\hline 12 & 20 & 0 & 0 & 10 & 20 & 20 \\
\hline 13 & 10 & 60 & 60 & 60 & 60 & 40 \\
\hline 14 & 100 & 60 & 70 & 60 & 60 & 50 \\
\hline Overall & 36.43 & 35 & 35.71 & 38.57 & 40.71 & 37.14 \\
\hline
\end{tabular}

Table 8: Precision in Top-10 for definition-3 of a "hit"

For this definition of a hit, our algorithm performed better than the baseline for three out of five different ' $\alpha$ '. These cases are M3, M4 and M5. Among these M4 had the 
best performance. When we compare M4 versus the baseline, M4 has done better than or equal to the baseline for nine out of the 14 queries.

In the table below, we look at the results for precision in top three.

\begin{tabular}{|c|c|c|c|c|c|c|}
\hline Query\# & M0 & M1 ( $\alpha=1)$ & M2 ( $\alpha=0.75)$ & M3 ( $\alpha=0.5)$ & M4 ( $\alpha=0.25)$ & M5 $(\alpha=0)$ \\
\hline & & & & & & \\
\hline 1 & 67 & 33 & 33 & 33 & 67 & 67 \\
\hline 2 & 0 & 67 & 100 & 100 & 100 & 33 \\
\hline 3 & 100 & 67 & 67 & 67 & 33 & 33 \\
\hline 4 & 0 & 33 & 33 & 33 & 67 & 33 \\
\hline 5 & 33 & 33 & 33 & 33 & 33 & 33 \\
\hline 6 & 67 & 33 & 33 & 33 & 33 & 67 \\
\hline 7 & 67 & 33 & 67 & 33 & 33 & 33 \\
\hline 8 & 0 & 67 & 67 & 67 & 67 & 67 \\
\hline 9 & 0 & 0 & 33 & 67 & 67 & 67 \\
\hline 10 & 33 & 33 & 67 & 67 & 33 & 33 \\
\hline 11 & 100 & 67 & 67 & 67 & 100 & 100 \\
\hline 12 & 0 & 0 & 0 & 33 & 33 & 33 \\
\hline 13 & 0 & 33 & 33 & 33 & 0 & 0 \\
\hline 14 & 100 & 100 & 100 & 100 & 67 & 67 \\
\hline Overall & 40.5 & 42.79 & 52.36 & 54.71 & 52.36 & 47.57 \\
\hline
\end{tabular}

Table 9: Precision in Top-3 for definition-3 of a "hit"

It can be see from the results in the above table that our algorithm has performed better than the baseline for all the values of ' $\alpha$ '. The best performance was obtained in the case of M3 where ' $\alpha$ ' $=0.5$. Again, 3 out of 4 cases that actually took the parent-child relationships into account performed better than M5, where only the concept vectors were used. Also, for nine out of 14 queries, M3 has performed better than or equal to the baseline.

\subsubsection{Proof of significance and overall discussion}


From the results in the above sections, we conclude that our matching algorithm has performed better than the baseline for almost all the different values of ' $\alpha$ ' and for all the definitions of "hit". The method M4 where ' $\alpha$ ' $=0.25$ performed the best in all but one case. We also observe that the cases that made use of the hierarchical relationships between the concepts performed better than the case M5, which just used the concept vectors. This improvement is more pronounced when we looked at the precision in top 3 results. Ranking plays a very important role in the case of related content because users are generally not interested beyond the top 3-5 results. In order to verify the statistical significance of our findings, we conducted a twotailed t-test between the results for the baseline, M4 and M5. The outcome of a t-test gives us the probability ' $p$ ' that the null hypothesis is true. The null hypothesis states that the difference or improvement observed is due to randomness. The critical value of ' $\mathrm{p}$ ' is generally taken as 0.05 . So, $\mathrm{p}<0.05$ means that there is less 5 percent chance that the difference was due to randomness and the null hypothesis can safely be rejected. In the following three tables, we report the results of the t-test for the three comparisons we are interested in. These are the baseline M0 (keyword vectors) versus M4 (best performing method that made use of concept trees), M0 versus M5 (the method which just made use of just the concept vectors) and also M4 versus M5.

\begin{tabular}{|c|c|c|c|c|c|c|c|c|}
\hline $\begin{array}{l}\text { DEFINITION OF } \\
\text { "HIT" }\end{array}$ & \multicolumn{4}{|c|}{ PRECISION TOP 10} & \multicolumn{4}{|c|}{ PRECISION TOP 3} \\
\hline & $\begin{array}{l}\text { Mean for } \\
\text { M0 }\end{array}$ & $\begin{array}{l}\text { Mean for } \\
\text { M4 }\end{array}$ & $\begin{array}{c}\text { Improvement } \\
(\%)\end{array}$ & p value & $\begin{array}{c}\text { Mean for } \\
\text { M0 }\end{array}$ & $\begin{array}{l}\text { Mean for } \\
\text { M4 }\end{array}$ & $\begin{array}{c}\text { Improvement } \\
(\%)\end{array}$ & p value \\
\hline At least 2 of 3 & 47.14 & 61.43 & 30.31 & 0.02 & 50 & 71.5 & 43 & 0.11 \\
\hline At least 1 of 3 & 77.86 & 90 & 15.59 & 0.05 & 71.21 & 92.71 & 30.19 & 0.02 \\
\hline Average Score $>=1$ & 36.43 & 40.71 & 11.75 & 0.53 & 40.5 & 52.36 & 29.28 & 0.38 \\
\hline
\end{tabular}

Table 10: p values for M0 versus M4 


\begin{tabular}{||l|c|c|c|c|c|c|c|c||}
\hline $\begin{array}{c}\text { DEFINITION OF } \\
\text { "HIT" }\end{array}$ & \multicolumn{4}{|c|}{ PRECISION TOP 10 } & \multicolumn{5}{c||}{ PRECISION TOP 3 } \\
\hline & $\begin{array}{c}\text { Mean for } \\
\text { M0 }\end{array}$ & $\begin{array}{c}\text { Mean for } \\
\text { M5 }\end{array}$ & $\begin{array}{c}\text { Improvement } \\
(\%)\end{array}$ & p value & $\begin{array}{c}\text { Mean for } \\
\text { M0 }\end{array}$ & $\begin{array}{c}\text { Mean for } \\
\text { M5 }\end{array}$ & $\begin{array}{c}\text { Improvement } \\
(\%)\end{array}$ & p value \\
\hline At least 2 of 3 & 47.14 & 60.71 & 28.79 & $\mathbf{0 . 0 3}$ & 50 & 69.07 & 38.14 & 0.09 \\
\hline At least 1 of 3 & 77.86 & 89.29 & 14.68 & $\mathbf{0 . 0 5}$ & 71.21 & 90.29 & 26.79 & $\mathbf{0 . 0 1}$ \\
\hline Average Score >=1 & 36.43 & 37.14 & 1.95 & 0.91 & 40.5 & 47.57 & 17.46 & 0.49 \\
\hline
\end{tabular}

Table 11: p values for M0 versus M5

\begin{tabular}{||l|c|c|c|c|c|c|c|c||}
\hline \hline $\begin{array}{c}\text { DEFINITION OF } \\
\text { "HIT" }\end{array}$ & \multicolumn{4}{|c|}{ PRECISION TOP 10 } & \multicolumn{5}{c||}{ PRECISION TOP 3 } \\
\hline & $\begin{array}{c}\text { Mean for } \\
\text { M5 }\end{array}$ & $\begin{array}{c}\text { Mean for } \\
\text { M4 }\end{array}$ & $\begin{array}{c}\text { Improvement } \\
(\%)\end{array}$ & p value & $\begin{array}{c}\text { Mean for } \\
\text { M5 }\end{array}$ & $\begin{array}{c}\text { Mean for } \\
\text { M4 }\end{array}$ & $\begin{array}{c}\text { Improvement } \\
(\%)\end{array}$ & p value \\
\hline At least 2 of 3 & 60.71 & 61.43 & 1.19 & 0.81 & 69.07 & 71.5 & 3.52 & 0.71 \\
\hline At least 1 of 3 & 89.29 & 90 & 0.8 & 0.34 & 90.29 & 92.71 & 2.68 & 0.58 \\
\hline Average Score >= 1 & 37.14 & 40.71 & 9.61 & 0.06 & 47.57 & 52.36 & 10.07 & 0.44 \\
\hline \hline
\end{tabular}

Table 12: $p$ values for M5 versus M4

It can be seen from the above tables that the methods that made use of concepts performed significantly better than the baseline for two of our three definitions of a good recommendation or "hit". Although the method that made use of the concept trees performed better than the one that only used the concept vectors, the difference was not significant. We believe that this is because of the size of the taxonomy that we used. It had only 368 categories spread across just three levels. We also used the top 20 categories obtained from the classifier for each document. These could have been concentrated in a single section of the taxonomy because it had only three levels. We used this taxonomy because it was appropriate for our dataset. We believe that in the case of bigger taxonomies with more number of levels, our concept tree 
method will definitely give better results than what we have observed here. We plan to conduct more experiments in that direction.

\section{Chapter 5 - Conclusions and Future Work}

\subsection{Conclusions}

In this work, we presented a new approach to compute document similarity that is based on a tree-matching algorithm. Unlike some of the traditional methods that match documents syntactically, our method matches documents conceptually. We discussed the process of constructing concept trees for documents using the concept vectors obtained from a classifier. We also presented an algorithm to find similarity between these concept trees that is based on tree edit distance algorithm.

To measure the effectiveness of our new approach, we conducted a user study. In this study, we compared our approach against the traditional text similarity based approach. The results of this study proved that our approach performed significantly better than the text similarity based approach. We observed that we got the best results when we used a weight propagation factor of 0.25 during the tree building stage. The weight propagation factor is used to propagate weights from the child to 
the parent categories. We also observed that our concept tree method performed better than the method that made use of only the concept vectors (i.e. a weight propagation factor of zero). But the improvement was not significant. We discussed the reasons for this and plan to conduct some further experiments based on our observations.

\subsection{Future Work}

There are a lot different ways in which our work can be used or extended. In this section we list some directions for future work.

- Although we presented our work in the context of text documents, it can easily be extended to other objects for which conceptual information is available. For example, it can be used for products in an ecommerce Website that can be classified into different categories.

- We used the tree edit distance algorithm to match our concept trees. It will be interesting to see how other tree-matching algorithms like those based on Tree Isomorphism perform in finding similarities between these concept trees.

- It will also be interesting to see how our method can be combined with one or more of the other methods to compute similarity between different objects.

- Our concept trees are derived from the same taxonomy. Some modifications to our algorithm would be needed to apply it in the case of concept trees that 
come from two different taxonomies. It will be interesting to see how it will perform in that situation.

- A recommender system that makes use of our algorithm to generate recommendations can be implemented. It will be straightforward to do it in the case of CiteSeer because we already used its data for our experiments and found that our algorithm works well with it. 


\section{References}

[1] A. Joshi and R. Krishnapuram. On Mining Web Access Logs. In Proceedings of the

2000 ACM SIGMOD Workshop on Research Issues in Data Mining and Knowledge Discovery 2000, pp. 63--69, 2000.

[2] A. Nierman and H. V. Jagadish. "Evaluating Structural Similarity in XML Documents". In Int'l Workshop on the Web and Databases (WebDB), Madison,WI, Jun. 2002.

[3] Bawa, M., Condie, T., and Ganesan, P. 2005. LSH forest: self-tuning indexes for similarity search. In Proceedings of the 14th international Conference on World Wide Web (Chiba, Japan, May 10 - 14, 2005). WWW '05. ACM Press, New York, NY, 651-660.

[4] Bollacker, K. D., Lawrence, S., and Giles, C. L. 1998. CiteSeer: an autonous Web agent for automatic retrieval and identification of interesting publications. In Proceedings of the Second international Conference on Autonomous Agents (Minneapolis, Minnesota, United States, May 10 - 13, 1998). K. P. Sycara and M. Wooldridge, Eds. AGENTS '98. ACM Press, New York, NY, 116-123.

[5] Chen, W. New algorithm for ordered tree-to-tree correction problem. Journal of Algorithms, 40:135-158, 2001.

[6] Deerwester, S., Dumais, S. T., Furnas, G. W., Landauer, T. K., \& Harshman, R. (1990). Indexing by latent semantic analysis. Journal of the American Society for Information Science, 41(6), 391-407.

[7] G. Valiente. Algorithms on Trees and Graphs. Springer-Verlag, Berlin, 2002.

[8] Ganesan, P., Garcia-Molina, H., and Widom, J. 2003. Exploiting hierarchical domain structure to compute similarity. ACM Trans. Inf. Syst. 21, 1 (Jan. 2003), 6493. 
[9] Henry Small. Co-citation in scientific literature: A new measure of the relationship between two documents. Journal of the American Society for Information Science, 24:265-269, 1973.

[10] http://acm.org/class/1998/

[11] http://citeseer.ist.psu.edu

[12] http://money.cnn.com/magazines/fortune/fortune_archive/2006/11/27/8394347/? postversion $=2006112010$

[13] Jeh, G. and Widom, J. 2002. SimRank: a measure of structural-context similarity. In Proceedings of the Eighth ACM SIGKDD international Conference on Knowledge Discovery and Data Mining (Edmonton, Alberta, Canada, July 23 - 26, 2002). KDD '02. ACM Press, New York, NY, 538-543.

[14] K. Tai, 'The tree-to-tree correction problem', J. ACM, 26, (3), 422-433 (1979).

[15] K. Zhang and D. Shasha, Simple fast algorithms for the editing distance between trees and related problems, SIAM J. Computing, 18, (6), 1245-1262 (1989).

[16] Reis, D. C., Golgher, P. B., Silva, A. S., and Laender, A. F. 2004. Automatic Web news extraction using tree edit distance. In Proceedings of the 13th international Conference on World Wide Web (New York, NY, USA, May 17 - 20, 2004). WWW '04. ACM Press, New York, NY, 502-511.

[17] Rubner, Y., Tomasi, C., and Guibas, L. J. 2000. The Earth Mover's Distance as a Metric for Image Retrieval. Int. J. Comput. Vision 40, 2 (Nov. 2000), 99-121.

[18] S. Lu, 'A tree-to-tree distance and its application to cluster analysis', IEEE Trans. Pattern Analysis and Machine Intelligence, PAMI-1, (2), 219-224 (1979).

[19] S. M. Selkow, 'The tree-to-tree editing problem', Information Processing Letters, 6, (6), 184-186 (1977).

[20] Sager, T., Bernstein, A., Pinzger, M., and Kiefer, C. 2006. Detecting similar Java classes using tree algorithms. In Proceedings of the 2006 international Workshop on Mining Software Repositories (Shanghai, China, May 22 - 23, 2006). MSR '06. ACM Press, New York, NY, 65-71.

[21] Sahami, M. and Heilman, T. D. 2006. A Web-based kernel function for measuring the similarity of short text snippets. In Proceedings of the 15th international Conference on World Wide Web (Edinburgh, Scotland, May 23 - 26, 2006). WWW '06. ACM Press, New York, NY, 377-386.

[22] SALTON, G., ANDBUCKLEY, C. Term weighting approaches in automatic 
text retrieval". Tech Report 87-881 Dept. of Computer Science, Cornell University, 1997.

[23] Susan Gauch, Juan M. Madrid, Subhash Induri, Devanand Ravindran, and Sriram Chadlavada, KeyConcept: A Conceptual Search Engine, Information and Telecommunication Technology Center, Technical Report: ITTC-FY2004-TR-864637, University of Kansas.

[24] Wan, X. and Peng, Y. 2005. The earth mover's distance as a semantic measure for document similarity. In Proceedings of the 14th ACM international Conference on information and Knowledge Management (Bremen, Germany, October 31 November 05, 2005). CIKM '05. ACM Press, New York, NY, 301-302.

[25] Xi, W., Fox, E. A., Fan, W., Zhang, B., Chen, Z., Yan, J., and Zhuang, D. 2005. SimFusion: measuring similarity using unified relationship matrix. In Proceedings of the 28th Annual international ACM SIGIR Conference on Research and Development in information Retrieval (Salvador, Brazil, August 15 - 19, 2005). SIGIR '05. ACM Press, New York, NY, 130-137.

[26] Yang, W. 1991. Identifying syntactic differences between two programs. Softw. Pract. Exper. 21, 7 (Jul. 1991), 739-755.

[27] Zhai, Y. and Liu, B. 2005. Web data extraction based on partial tree alignment. In Proceedings of the 14th international Conference on World Wide Web (Chiba, Japan, May 10 - 14, 2005). WWW '05. ACM Press, New York, NY, 76-85.

[28] Suzuki, N. 2005. Finding an optimum edit script between an XML document and a DTD. In Proceedings of the 2005 ACM Symposium on Applied Computing (Santa Fe, New Mexico, March 13 - 17, 2005). L. M. Liebrock, Ed. SAC '05. ACM Press, New York, NY, 647-653.

[29] Xing, G., Malla, C. R., Xia, Z., and Venkata, S. D. 2006. Computing edit distances between an XML document and a schema and its application in document classification. In Proceedings of the 2006 ACM Symposium on Applied Computing (Dijon, France, April 23 - 27, 2006). SAC '06. ACM Press, New York, NY, 831-835. 